\title{
Incertitudes sur les expositions, les niveaux de contamination et les doses après inhalation d'actinides
}

\author{
P. FRITSCH ${ }^{1}$, P. RAYNAUD ${ }^{2}$, N. BLANCHIN ${ }^{3}$ et A. MIÈLE ${ }^{3}$
}

(Manuscrit reçu le 12 décembre 2005, accepté le 20 avril 2006)

RÉSUMÉ Dans l'industrie nucléaire française, les doses engagées après inhalation de radionucléides sont généralement évaluées à partir de mesures biologiques dont l'analyse peut permettre de remonter (démarche ascendante) à l'incorporation initiale puis à la dose par application de la dose par unité d'exposition (DPUI). Une valeur spécifique de la DPUI peut être attribuée à chaque contamination. Cet article présente une synthèse des données de la littérature et de résultats de recherches en cours concernant les incertitudes sur l'estimation de l'incorporation, de la biocinétique des radionucléides et sur les doses après une exposition à des aérosols renfermant des actinides. Une comparaison des incertitudes a été réalisée après une application ascendante et descendante des modèles. Dans ce dernier cas, la contamination interne est évaluée à partir de prélèvements atmosphériques effectués au poste de travail. Les incertitudes les plus élevées sont calculées après une application descendante des modèles. L'interprétation des différents résultats qui sont ici rapportés souligne l'intérêt de la connaissance des incertitudes pour évaluer de manière réaliste l'impact dosimétrique d'une contamination accidentelle. Par ailleurs, différents exemples illustrent les limites de l'application de certains modèles de la Commission internationale de protection radiologique (CIPR) qui sont utilisés en radioprotection. Au-delà, cette synthèse souligne les difficultés de l'estimation du risque de cancer à partir d'un calcul de doses, notamment, du fait de l'hétérogénéité de leur distribution au sein des différentes régions cible du tractus respiratoire.

ABSTRACT Uncertainties in exposures, contamination level and doses after inhalation of actinides.

In France, after occupational exposure to radioactive aerosols, committed doses are currently calculated by an ascending approach from biological data to estimate the initial contamination using dose per unit intake (DPUI). Specific DPUI can be calculated depending on the contamination. This paper is an overview on uncertainties in exposure level, biokinetics of radionuclides and doses after inhalation exposure to aerosol containing actinides. Data reported in the literature and those obtained in studies which are still in progress are described. A comparison of the uncertainties has been done after an ascending and a descending approach. In this later case, aerosol deposition within the respiratory tract is estimated from air sampling at the work place (estimate of the activity of the aerosol). The largest uncertainties were calculated after a descending application of the models. The results here reported pointed out the need of the knowledge of the uncertainties for

CEA, Laboratoire de radiotoxicologie, SRCA/DRR/DSV, B.P. 12, 91680 Bruyères-le-Châtel, France.

Service de santé au travail, COGEMA-Marcoule, 30207 Bagnols-sur-Cèze, France.

CEA, Service de santé au travail, Cadarache, 13108 Saint-Paul-Lez-Durance, France. 
a realistic interpretation of the results obtained after dose calculation, as well as the application limits of some ICRP models. Moreover, difficulties to estimate risk of cancer from doses are pointed out, which are mainly associated with the heterogeneity of the distribution of alpha dose within the different target regions of the respiratory tract.

Keywords: uncertainty / aerosol / actinide / dosimetry / inhalation

\section{Introduction}

L'inhalation constitue l'un des principaux modes de contamination interne par des radionucléides, notamment au cours de la fabrication du combustible utilisé dans les centrales électronucléaires. Dans ce cadre, différentes mesures radiologiques sont prises pour assurer le suivi sanitaire des travailleurs. La surveillance en continu de l'atmosphère aux postes de travail est assurée par des balises qui détectent rapidement une présence anormale d'aérosols radioactifs (seuil de détection alpha des actinides : $0,5 \mathrm{~Bq}$ ). Ces équipements mesurent également l'activité atmosphérique cumulée sur 24-48 heures, ou d'avantage, permettant d'évaluer le bruit de fond des installations. Parallèlement, le suivi des travailleurs est effectué par des mesures anthroporadiamétriques et radiotoxicologiques sur les excrétas réalisées de façon régulière avec une fréquence dépendant de la période efficace du radioélément considéré.

Pour les actinides, l'excrétion fécale est généralement très supérieure à l'excrétion urinaire et permet de détecter une contamination par inhalation, mais, en l'absence d'autres indicateurs d'exposition, l'interprétation des résultats est particulièrement délicate (Blanchin et al., 2005). Les limites de détection des différentes mesures sont de l'ordre de $10 \mathrm{~Bq} \mathrm{~d}{ }^{241} \mathrm{Am}$ pour les comptages thoraciques $\gamma$, (radionucléide contribuant à 5-10\% de l'activité alpha totale des MOX en cours de fabrication), et de respectivement 1 et $0,2 \mathrm{mBq}$ pour l'activité alpha présente dans les excrétions fécales et urinaires journalières. En fait, dans le cas du MOX, la présence $\mathrm{d}^{241} \mathrm{Am}$ améliore considérablement la limite de détection de l'activité thoracique car le seuil de détection des raies $\mathrm{X} \mathrm{du}{ }^{238-239} \mathrm{Pu}$ est supérieur à $1 \mathrm{kBq}$.

L'ensemble de cette démarche a pour but, après un calcul de la dose efficace, de s'assurer que l'exposition annuelle des travailleurs est inférieure à la limite réglementaire de $20 \mathrm{mSv}$ cumulés pour les irradiations externe et interne. On peut définir une limite annuelle «d'incorporation » (LAI) par l'activité d'un aérosol qui, après inhalation, conduit à une dose efficace engagée sur 50 ans égale à $20 \mathrm{mSv}$ ). Dans le cas de contaminations significatives, un suivi radiologique de chaque individu est assuré. 
Les doses sont généralement obtenues en utilisant les modèles de la Commission internationale de protection radiologique (CIPR), en premier lieu de manière ascendante, pour ajuster au mieux des prévisions théoriques avec les données biologiques, afin d'évaluer l'activité de l'aérosol responsable de la contamination. Dans cette approche, des paramètres de dissolution propres au radiocontaminant peuvent être évalués. Enfin, la dose efficace est calculée en utilisant une dose par unité d'exposition (en anglais, Dose Per Unit Intake : DPUI) standard ou spécifique. Différents outils (logiciels) sont disponibles pour faciliter l'application ascendante des modèles de la CIPR dont plusieurs ont fait récemment l'objet d'une revue (Ansoborlo et al., 2003). Aujourd'hui, aucune incertitude n'est associée au résultat du calcul de dose. Néanmoins, a priori, les incertitudes sur les dépôts thoraciques, et donc sur les doses, seraient plus faibles lorsqu'ils sont mesurés à partir de l'anthroporadiamétrie, qu'à partir de l'évaluation de l'activité des aérosols prélevés aux postes de travail. Dans ce dernier cas, les modèles sont appliqués de manière descendante, en utilisant les DPUI proposées par défaut. En pratique, dans la mesure où des données biologiques sont disponibles, il apparaît que l'application descendante des modèles tend à surestimer les niveaux de contamination interne (Blanchin et al., 2004).

Depuis plus de 10 ans, la CIPR a évalué la variabilité de la valeur de certains paramètres introduits dans les modèles (ICRP, 1994). Cependant, elle n'est pas parvenue, après inhalation, à caractériser l'impact de cette variabilité en termes d'incertitudes sur les niveaux de contamination et sur les doses. Parallèlement, différentes démarches ont été initiées afin d'évaluer les incertitudes sur les DPUI après ingestion, notamment d'iode radioactif, mais aussi de ${ }^{137} \mathrm{Cs}$ et de ${ }^{90} \mathrm{Sr}$, en tenant compte de la variabilité des différents paramètres des modèles (Dunning et Schwarz, 1980 ; Hamby et Benke, 1999; Harvey et al., 2003 ; Fritsch et al., 2003a, 2003b ; Apostoaei et Miller, 2004). En revanche, pour l'inhalation, les études sur les incertitudes sont limitées et les résultats obtenus demeurent encore trop fragmentaires pour être applicables en radioprotection.

Cet article constitue une synthèse des données de la littérature et de certaines études en cours concernant les incertitudes sur les expositions, les niveaux de contamination, la biocinétique des radionucléides et les doses, après inhalation d'oxydes d'actinides. Ce travail a été réalisé, en partie, dans le cadre du groupe de travail MEDOR (MEthodes DOsimétriques de Référence) qui a pour but, notamment, de proposer des démarches pertinentes pour l'évaluation des doses après contamination interne, en tenant compte des limites d'application de différents modèles. 


\section{Calcul et expression des incertitudes}

La variabilité de la valeur des paramètres introduits dans les modèles peut être décrite par différentes fonctions de densité de probabilité : uniformes, triangulaires, normales, log normales... À partir de ces fonctions, des simulations sont réitérées à de nombreuses reprises en attribuant des valeurs équiprobables à chacun des paramètres pour permettre une approche de type Monte Carlo. Elles correspondent à différents aérosols et différents individus ayant une anatomie et une physiologie spécifique. L'analyse de la distribution des résultats obtenus permet de définir des incertitudes qui sont souvent exprimées sous la forme d'intervalle de confiance à $95 \%$. Dans le cas d'une distribution normale, la valeur $97,5 \%$ est égale à la moyenne plus 2 écarts types $(\sigma)$ et la valeur $2,5 \%$ à la moyenne moins $2 \sigma$. Ainsi, $4 \sigma$, exprimé en valeur relative par rapport à la moyenne, correspond au rapport entre ces 2 valeurs. En revanche, dans le cas d'une distribution log-normale, ce rapport est égal à l'écart type géométrique $\left(\sigma_{\mathrm{g}}\right)$ à la puissance 4 , la valeur 97,5\% étant égale à la valeur médiane multipliée par $\sigma_{\mathrm{g}}$ au carré.

Dans la pratique, les distributions sont caractérisées, après au moins 1000 simulations, par une analyse graphique effectuée selon la méthode de Henry. Cette dernière permet de représenter toute loi normale ou log-normale sous forme de droite, et donc le choix de la distribution s'effectue en fonction de la valeur du coefficient linéaire de corrélation. Les distributions sont caractérisées comme normale ou log-normale lorsque la valeur de ce coefficient est supérieure à 0,98 (Fritsch, 2004).

Les résultats précédents sont obtenus en faisant varier simultanément la valeur des différents paramètres des modèles. Il est également possible d'évaluer l'impact de la variation de chacun des paramètres, ce qui constitue une étude de sensibilité. Cette approche permet d'identifier les paramètres dont la valeur doit être précisée afin de limiter de manière significative les incertitudes sur les doses.

\section{Incertitudes sur la taille des aérosols}

Un aérosol est caractérisé par la loi de distribution du diamètre de ses particules par rapport à leur radioactivité ou à leur effectif total. Ce diamètre peut être exprimé sous forme de diamètre aérodynamique $\left(\mathrm{d}_{\mathrm{ae}}\right)$ ou thermodynamique $\left(\mathrm{d}_{\mathrm{th}}\right)$. La relation entre ces deux diamètres est fonction de la forme et de la densité des particules (facteur de forme compris entre 1 et 2 , par défaut 1,5 et $3 \mathrm{~g} \mathrm{~cm}^{3}$ ). Schématiquement, le $\mathrm{d}_{\mathrm{ae}}$ reflète la vitesse de chute des particules alors que le $\mathrm{d}_{\mathrm{th}}$ reflète leur taille géométrique. Le $\mathrm{d}_{\mathrm{ae}}$ est utilisé pour le calcul du dépôt par impaction et sédimentation, majoritaire pour les particules de plus de $1 \mu \mathrm{m}$, alors 


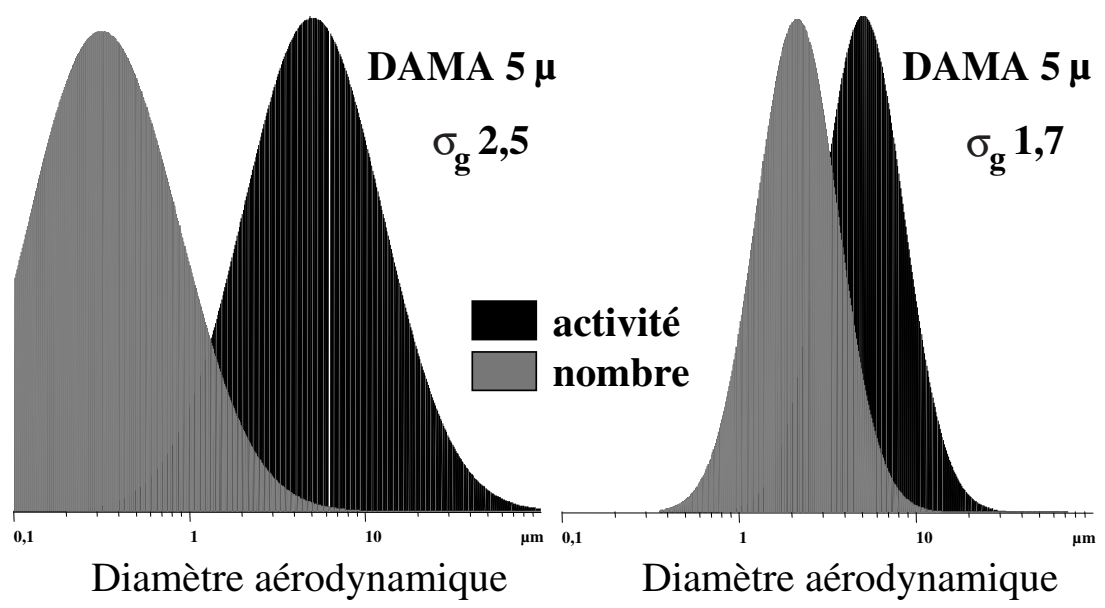

Figure 1 - Distributions en nombre et en activité des particules d'un aérosol ayant un DAMA de 5 Mm (densité 3, facteur de forme 1,5) avec un $\sigma_{g}$ de 2,5 (valeur par défaut) ou de 1,7.

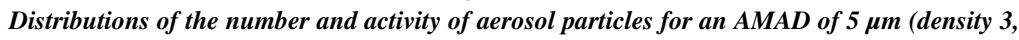
shape factor 1.5) with a $\sigma_{g}$ of 2.5 (default value) or 1.7.

que le $\mathrm{d}_{\text {th }}$ est utilisé pour le calcul du dépôt par diffusion brownienne, majoritaire pour des $\mathrm{d}_{\mathrm{ae}}$ de moins de $0,1 \mu \mathrm{m}$. La figure 1 compare les distributions en activité et en nombre des $\mathrm{d}_{\mathrm{ae}}$ pour le diamètre aérodynamique médian en activité (DAMA) appliqué par défaut en milieu industriel $\left(5 \mu \mathrm{m}, \sigma_{\mathrm{g}}=2,5\right)$. Elle montre également ces distributions pour un $\sigma_{\mathrm{g}}$ de 1,7 , valeur qui a pu être mesurée à certains postes de travail (Fritsch et al., 2006a).

Le DAMA n'est pas représentatif de la taille des aérosols bien qu'il lui soit directement lié à partir du moment où $\sigma_{\mathrm{g}}$ est défini. En effet, par défaut, seul 0,2\% des particules ont un $\mathrm{d}_{\mathrm{ae}}$ supérieur ou égal à $5 \mu \mathrm{m}$. La valeur du $\sigma_{\mathrm{g}}$ influe considérablement sur le nombre de particules par $\mathrm{Bq}$ d'aérosol. Ainsi, par exemple, pour le ${ }^{239} \mathrm{PuO}_{2}$ de DAMA $5 \mu \mathrm{m}$, un aérosol ayant un $\sigma_{\mathrm{g}}$ de 2,5 renferme environ 400 particules $\mathrm{Bq}^{-1}$, alors que lorsque $\sigma_{\mathrm{g}}$ est égal à 1,7 , il n'en renferme que $30 \mathrm{~Bq}^{-1}$. La valeur limite du $\sigma_{\mathrm{g}}$ est égale à 1 . Dans le cas où $\sigma_{\mathrm{g}}$ est proche de 1 , la taille moyenne des aérosols est voisine du DAMA. On parle alors d'aérosols monodispersés.

L'utilisation d'impacteurs en cascade a permis une évaluation directe du DAMA des oxydes d'actinides en milieu industriel. Cependant, les prélèvements ont été effectués essentiellement dans des boîtes à gants, ce qui n'est pas a priori comparable aux situations d'exposition professionnelle (Dorrian et Bailey, 1995 ; 
P. FRITSCH et al.

Ansoborlo et al., 2002). Pour les oxydes d'actinides peu solubles, les DAMA sont compris entre 3 et $8,5 \mu \mathrm{m}$ avec un $\sigma_{\mathrm{g}}$ souvent inférieur à la valeur par défaut de 2,5. Néanmoins, en cas d'incident, les aérosols peuvent également résulter de la remise en suspension de particules déposées sur différentes surfaces. Dans ce cas, leur agrégation pourrait entraîner une augmentation significative de la taille des aérosols.

Des études sont en cours sur l'évaluation de la taille des aérosols, durant la fabrication du combustible MOX, par analyse autoradiographique des filtres des balises de surveillance atmosphérique selon un protocole précédemment décrit (Ramounet-Le Gall et al., 2002; Fritsch et Guillet, 2002). Pour des filtres « positifs » $(>0,5 \mathrm{~Bq})$, cette méthode s'avère particulièrement sensible au poste de la rectification. Dans ce cas, pour plus de la moitié des incidents étudiés, le DAMA apparaît supérieur à $10 \mu \mathrm{m}(\min 4 \mu \mathrm{m}, \max 50 \mu \mathrm{m})$. Des résultats analogues ont été obtenus en amont de la rectification, mais, les mesures sont alors plus approximatives, du fait de la présence d'un mélange de particules d' $\mathrm{UO}_{2}$ et de $\mathrm{PuO}_{2}$. Cette étude montre, qu'en cas d'incident, le DAMA apparaît plus dépendant des circonstances de la contamination que de la nature du poste de travail (Fritsch et al., 2006a); d'où l'intérêt d'une évaluation systématique du DAMA en cas d'incident entraînant une contamination interne significative. En effet, pour des composés peu solubles tels les oxydes d'uranium et/ou de plutonium, la valeur par défaut des DPUI varie considérablement avec la taille des aérosols. Par exemple, pour le ${ }^{239} \mathrm{PuO}_{2}$, elle diminue graduellement de 15 à 8,3 et à $5,9 \mu \mathrm{Sv} \mathrm{Bq}^{-1}$ pour des DAMA de 1 , de 5 et de $10 \mu \mathrm{m}$.

\section{Incertitudes sur le dépôt des aérosols}

Le modèle du dépôt des aérosols de la publication 66 de la CIPR (ICRP, 1994) considère 5 principales régions : le nez antérieur (ET1) et les autres régions extra thoraciques, du nez postérieur au larynx (ET2), l'arbre trachéo bronchique (BB), les bronchioles (bb) et les alvéoles (AI). Des sous régions sont également définies : les régions séquestrées, où les particules ayant traversé l'épithélium sont localisées dans les macrophages de l'interstitium (ETseq, BBseq et bbseq), les dépôts à la surface du mucus ou dans le mucus, qui sont rapidement épurés (BB1 et bb1), et les dépôts entre le mucus et la surface cellulaire, qui sont épurés plus lentement (BB2 et bb2). Par ailleurs, selon la vitesse de l'épuration mécanique, AI est divisé en 3 sous régions (AI1, AI2 et AI3). Le modèle de la CIPR assimile les principales régions du tractus respiratoire à des filtres successifs, durant l'inhalation et l'exhalation des aérosols. De très nombreux paramètres sont introduits dans ce modèle, et leur variabilité a été précédemment évaluée en ce qui concerne :

1. les propriétés physiques des aérosols, tels, le $\sigma_{\mathrm{g}}$, la densité et la forme des particules (Harvey et Hamby, 2001), 
TABLEAU I

Calcul et incertitudes sur les paramètres décrivant les propriétés physiques des aérosols, l'inhalabilité et l'efficacité des dépôts aéro et thermodynamiques. Valeurs rapportées par ${ }^{1}$ Harvey et Hamby (2001), ${ }^{2}$ Bloch et al. (2001) et la ${ }^{3}$ publication 60 de la CIPR (ICRP, 1994). Calculation and uncertainty in parameters describing physical properties of aerosols, inhalability and efficiency in thermo and aerodynamic deposition.

\begin{tabular}{|c|c|c|c|}
\hline Description des paramètres et mode de calcul & $\begin{array}{c}\text { Type de } \\
\text { distribution }\end{array}$ & $\begin{array}{l}\text { Moyenne } \\
\text { médiane } \\
\text { minimum }\end{array}$ & $\begin{array}{c}\sigma \\
\sigma_{\mathrm{g}} \\
\operatorname{maximum}\end{array}$ \\
\hline${ }^{1}$ Densité des particules (d) & triangulaire & $\begin{array}{c}1,0 \\
\text { mode } 3.0\end{array}$ & 10,0 \\
\hline${ }^{1}$ Facteur de forme $(\chi)$ & triangulaire & $\begin{array}{c}1,1 \\
\text { mode } 1,5\end{array}$ & 1,9 \\
\hline $\begin{array}{l}{ }^{2} \sigma_{\mathrm{g}} \text { de la distribution des } \mathrm{d}_{\mathrm{ae}} \text { en activité } \\
\sigma_{\mathrm{g}}=1+1,5\left(1-\left(100 \mathrm{DTMA} \mathrm{A}^{1,5}+1\right)^{-1}\right) \\
\mathrm{d}_{\text {th }} \text { médian en activité DTMA calculé à partir du DAMA }\end{array}$ & uniforme & $\begin{array}{c}\text { GSD } \times 0,7 \\
\text { ou } 1,0\end{array}$ & $\mathrm{GSD} \times 1,1$ \\
\hline $\begin{array}{l}{ }^{2} \text { Inhalabilité des particules: } \mathrm{I}_{1}=1-0,5\left(1-\left(7,6 \times 10^{-4} \mathrm{~d}_{\mathrm{ae}}{ }^{2,8}+1\right)^{-1}\right. \\
\text { Erreur relative } \mathrm{E}_{\mathrm{inh}} \text { pour } \mathrm{d}_{\mathrm{ae}}>0.5 \mu \mathrm{m} \text { sinon } \mathrm{E}_{\mathrm{inh}}=0\end{array}$ & normale & 1 & 0,1 \\
\hline \multicolumn{4}{|l|}{${ }^{3}$ Efficacité régionale des dépôts aero et thermodynamiques } \\
\hline $\mathrm{Cae}\left(\mathrm{ET}_{1}\right)$ & log-normale & 1 & 1,82 \\
\hline $\mathrm{Cae}\left(\mathrm{ET}_{2}\right)$ & log-normale & 1 & 1,82 \\
\hline $\mathrm{Cae}(\mathrm{BB})$ & log-normale & 1 & 1,58 \\
\hline $\mathrm{Cae}(\mathrm{bb})$ & log-normale & 1 & 1,58 \\
\hline $\mathrm{Cae}(\mathrm{AI})$ & log-normale & 1 & 1,30 \\
\hline $\mathrm{Cth}\left(\mathrm{ET}_{1}\right)$ & log-normale & 1 & 1,18 \\
\hline $\mathrm{Cth}\left(\mathrm{ET}_{2}\right)$ & log-normale & 1 & 1,18 \\
\hline Cth(BB) & log-normale & 1 & 1,23 \\
\hline $\mathrm{Cth}(\mathrm{bb})$ & log-normale & 1 & 1,23 \\
\hline $\mathrm{Cth}(\mathrm{AI})$ & log-normale & 1 & 1,23 \\
\hline
\end{tabular}

2. leur inhalabilité et l'efficacité de leurs dépôts aéro- et thermodynamiques au sein des principales régions du tractus respiratoire (ICRP, 1994),

3. l'anatomie, la physiologie et l'activité des individus (Bolch et al., 2001 ; Layton, 1993),

4. la répartition des dépôts parmi les différentes régions du tractus respiratoire (Bolch et al., 2003).

Par ailleurs, dans une gamme d'activités proches de 1 LAI, pour les composés de forte activité spécifique, le nombre total de particules présent dans l'aérosol constitue également une source importante d'incertitudes sur la valeur des dépôts (Fritsch, 2004). Le tableau I montre la variabilité qui est généralement utilisé pour différents paramètres décrivant les aérosols et leur dépôt thermo et aérodynamique au sein des différentes régions du tractus respiratoire. 


\section{TABLEAU II}

Calcul et incertitudes sur les paramètres décrivant l'anatomie et la physiologie des individus. Valeurs issues de ${ }^{1}$ Bloch et al. (2001) et de ${ }^{2}$ Fritsch (2006).

Calculation and uncertainty in parameters describing anatomy and physiology of individuals. Values from ${ }^{1}$ Bloch et al. (2001) and ${ }^{2}$ Fritsch (2006).

\begin{tabular}{|c|c|c|c|}
\hline Description des paramètres et mode de calcul & $\begin{array}{c}\text { Type de } \\
\text { distribution }\end{array}$ & $\begin{array}{l}\text { Moyenne } \\
\text { médiane } \\
\text { minimum }\end{array}$ & $\begin{array}{c}\sigma \\
\sigma_{\mathrm{g}} \\
\text { Maximum }\end{array}$ \\
\hline $\begin{array}{l}{ }^{1} \text { Age }(\mathrm{A}, \text { années }) \\
{ }^{1} \text { Taille }(\mathrm{Ht}, \mathrm{cm}) \\
{ }^{1} \text { Indice de masse corporelle }\left(\mathrm{BMI}, \mathrm{kg} \mathrm{m}^{-2}\right) \\
\text { Poids } \mathrm{Wt}=\mathrm{Ht} \times \mathrm{Ht} / \mathrm{BMI}\end{array}$ & $\begin{array}{l}\text { constant } \\
\text { normale } \\
\text { log-normale }\end{array}$ & $\begin{array}{c}30 \\
176,7 \\
24,89\end{array}$ & $\begin{array}{c}6,7 \\
1,17\end{array}$ \\
\hline 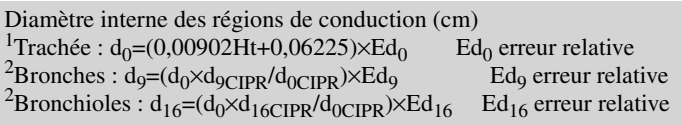 & $\begin{array}{l}\text { normale } \\
\text { normale } \\
\text { normale }\end{array}$ & $\begin{array}{l}1 \\
1 \\
1\end{array}$ & $\begin{array}{l}0,1 \\
0,1 \\
0,2\end{array}$ \\
\hline $\begin{array}{l}{ }^{1} \text { Volume mort total }(\mathrm{ml}): \mathrm{V}_{\mathrm{D}}=8,72 \cdot \exp (0,0162 \mathrm{Ht}) \mathrm{E}_{\mathrm{VD}} \\
{ }^{\mathrm{E}_{\mathrm{VD}} \text { erreur relative }} \\
{ }^{2} \mathrm{~V}_{\mathrm{D}}(\mathrm{ET})=\left(\mathrm{V}_{\mathrm{D}}(\mathrm{ET}){ }_{\mathrm{CIPR}} / \mathrm{d}_{0 \mathrm{CIPR}}{ }^{2}\right) \times \mathrm{d}_{0}{ }^{2} \times \mathrm{E}_{\mathrm{VD}} \quad \mathrm{V}_{\mathrm{D}}(\mathrm{BB})=\mathrm{V}_{\mathrm{D}}(\mathrm{bb})= \\
0,5\left(\mathrm{~V}_{\mathrm{D}}-\mathrm{V}_{\mathrm{D}}(\mathrm{ET})\right)\end{array}$ & log-normale & 1 & 1,17 \\
\hline $\begin{array}{l}{ }^{1} \text { Capacité fonctionnelle résiduelle }(\mathrm{mL}) \text { : } \\
\text { FRC }=23,48 \mathrm{Ht}+9,0 \mathrm{~A}-1093+\mathrm{E}_{\mathrm{FRC}} \\
\mathrm{E}_{\mathrm{FRC}} \text { erreur résiduelle }\end{array}$ & normale & 0 & 600,0 \\
\hline \multicolumn{4}{|l|}{$\begin{array}{l}{ }^{1} \text { Taux de ventilation }\left(\mathrm{mL} \min ^{-1}\right) \text { : } \\
\mathrm{V}_{\mathrm{E}}=\mathrm{V}_{\mathrm{Q}} \times \mathrm{V}_{\mathrm{O} 2} \quad \mathrm{~V}_{\mathrm{O} 2}=\left(0,694 \times \mathrm{BMRH}_{\mathrm{oxy}}\right) \times \mathrm{B}_{\text {mult }}\end{array}$} \\
\hline $\begin{array}{l}{ }^{1} \text { Rapport ventilatoire équivalent } \\
\mathrm{V}_{\mathrm{Q}} \text { distribution }\end{array}$ & log-normale & 26,4 & 1,16 \\
\hline $\begin{array}{l}{ }^{1} \text { Basal metabolic rate }\left(\mathrm{MJ} \mathrm{d}^{-1}\right): B M R=0,063 \times W t+2,896+E_{B M R} \\
E_{B M R} \text { erreur résiduelle de BMR }\end{array}$ & normale & 0,0 & 0,6702 \\
\hline${ }^{1}$ Volume d' $\mathrm{O}_{2}$ nécessaire pour produire $1 \mathrm{~kJ}(\mathrm{~L}) \mathrm{H}_{\mathrm{oxy}}$ & uniforme & 0,0476 & 0,0529 \\
\hline $\begin{array}{l}{ }^{2} \text { Facteur multiplicatif du métabolisme de base }\left(\mathrm{B}_{\text {mult }}\right) \\
\text { Assis } \\
\text { Exercice léger } \\
\text { Exercice fort }\end{array}$ & $\begin{array}{l}\text { uniforme } \\
\text { uniforme } \\
\text { uniforme }\end{array}$ & $\begin{array}{l}1,1 \\
2,0 \\
5,5\end{array}$ & $\begin{array}{l}1,4 \\
5,0 \\
8,5\end{array}$ \\
\hline $\begin{array}{l}{ }^{2} \text { Fréquence respiratoire }\left(\mathrm{min}^{-1}\right) \\
\text { assis } B_{f}=12 \times E_{B f} \text { exercice léger } B_{f}=20 \times E_{B f} \text { exercice fort } B_{f}=26 \times E_{B f} \\
E_{B f} \text { erreur relative }\end{array}$ & log-normale & 1 & 1,25 \\
\hline
\end{tabular}

Le tableau II montre la variabilité des paramètres anatomiques et physiologiques qui a été utilisée pour évaluer les incertitudes sur le dépôt des aérosols polydispersés chez le travailleur (Fritsch, 2006).

Pour un aérosol monodispersé, les incertitudes sur leurs dépôts demeurent limitées, sauf pour les régions les plus profondes (poumon profond $=\mathrm{AI}+\mathrm{bb}$ ), où elles augmentent considérablement avec la taille des aérosols, lorsqu'elle devient supérieure à $1 \mu \mathrm{m}$ (Bolch et al., 2001). Par exemple, pour un $\mathrm{d}_{\mathrm{ae}}$ de $5 \mu \mathrm{m}$, le $\sigma_{\mathrm{g}}$ de la distribution log-normale des dépôts dans AI et bb sont respectivement 2,3 et 2,4, alors que pour un DAMA de $10 \mu \mathrm{m}$ ils sont égaux à 6,9 et 5,6. Certaines données 
suggèrent que les incertitudes augmenteraient lorsque l'aérosol est polydispersé (Aden et Scott, 2003). Lorsque le DAMA est inférieur à $2 \mu \mathrm{m}$, la distribution des dépôts au sein du poumon profond correspond à une loi log-normale. En revanche, à partir de $5 \mu \mathrm{m}$, aucune loi simple ne permet de décrire la distribution de ces dépôts, et le rapport entre la valeur 97,5 et $2,5 \%$ de la distribution est de l'ordre de $10^{16}$. De telles incertitudes apparaissent irréalistes, et rendraient illusoire tout calcul de dose à partir de l'activité de l'aérosol. Il faut cependant souligner que, dans une gamme de DAMA proche de la valeur proposée par défaut, Aden et Scott (2003) obtiennent des valeurs de dépôts profonds inférieures à celles rapportées dans la publication 66 de la CIPR (ICRP, 1994).

Les études d'incertitudes peuvent être biaisées par le choix de la variabilité des paramètres, et par leur interdépendance. Pour le dépôt des aérosols, nous avons repris les variations précédemment décrites (Bolch et al., 2001). La seule modification concerne la variation de la fréquence respiratoire, et non du volume courant, car elle permet de faire varier de façon réaliste cette fréquence selon l'activité des individus. Des incertitudes similaires à celles précédemment rapportés pour des aérosols monodispersés (Bolch et al., 2001) ont été obtenues (Fritsch, 2006). Les incertitudes sur le dépôt d'aérosols polydispersés s'avèrent beaucoup plus faibles (Fritsch, 2006). Dans une gamme de DAMA entre 0,1 à $50 \mu \mathrm{m}$, la médiane des dépôts est égale aux valeurs de la publication 66 de la CIPR (ICRP, 1994). Jusqu'à $1 \mu \mathrm{m}$, le $\sigma_{\mathrm{g}}$ des dépôts au sein des différentes régions est voisin de 1,5. Il augmente ensuite graduellement en atteignant, pour un DAMA de $50 \mu \mathrm{m}$, une valeur de 2,3 dans AI, 2,9 dans bb, et 2,0 dans BB. Ces résultats s'expliquent du fait, qu'au-delà d'un $\mathrm{d}_{\mathrm{ae}}$ de $15 \mu \mathrm{m}$, il n'y a plus d'incertitudes sur les dépôts profonds, car ils sont nuls. En revanche, les incertitudes sur les dépôts au sein des régions extra thoraciques sont maximales pour des DAMA de $0,5-1 \mu \mathrm{m}$. Elles diminuent lorsque la taille des aérosols augmente, pour atteindre un plateau voisin de 1,2. Ces résultats, obtenus pour le travailleur standard (assis $3 \mathrm{~h}$, exercice léger $5 \mathrm{~h}$, respiration par le nez), ne tiennent pas compte du volume total d'air inhalé durant une journée de travail. En effet, on suppose que tous les individus ont été exposés à un aérosol renfermant $1 \mathrm{~Bq}$. Pour être plus réaliste, il faut donc corriger l'exposition par le volume d'air inhalé par chaque individu, qui varie selon son anatomie et sa physiologie. La figure 2 compare les $\sigma_{\mathrm{g}}$ des dépôts corrigés ou non par ce volume.

En fait, en cas d'incident, la durée de l'exposition est le plus souvent limitée, du fait du déclenchement des balises de surveillance ou d'une rupture de confinement brutale et évidente. Les paramètres par défaut ne sont adaptés qu'à une exposition chronique. Ainsi, en cas d'incident, il conviendrait de connaître des paramètres spécifiques aux individus exposés (respiration nez/bouche, type d'activité, anatomie, ...) afin de mieux estimer le dépôt des aérosols et de pouvoir 


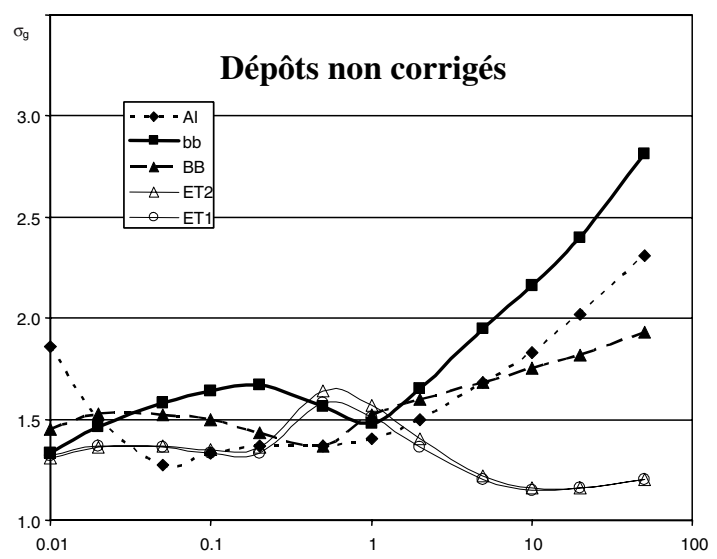

Diamètre aérodynamique $(\mu \mathrm{m})$

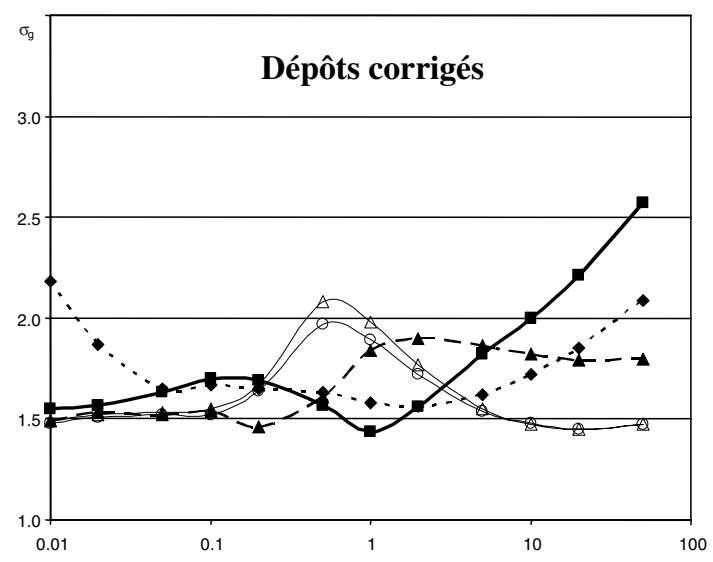

Diamètre aérodynamique $(\mu \mathrm{m})$

Figure 2 - Incertitudes sur le dépôt des aérosols au sein des principales régions du tractus respiratoire en fonction du DAMA. Les incertitudes, calculées pour le travailleur par défaut, sont exprimées sous forme de $\sigma_{g}$. Dépôts non corrigés : chaque individu a été exposé à une même activité, Dépôts corrigés : le volume d'air inspiré par chaque individu est pris en compte. D'après Fritsch (2006).

Uncertainties in aerosol deposition within the main regions of the respiratory tract of the standard worker expressed as $\sigma_{g}$ as a function of AMAD. "Dépôts non corrigés": each individual has been exposed to the same aerosol activity, "Dépôts corrigés": the volume of air inhaled by each individual is taken into account. From Fritsch (2006). 


\section{TABLEAU III}

Nombre moyen de particules déposées dans les différentes régions du tractus respiratoire avec son écart type qui augmente lorsque le nombre de particules présent dans l'aérosol diminue (DAMA $5 \mu \mathrm{m}, \sigma_{\mathrm{g}} 2,5$, densité 3, travailleur standard).

Mean number of particles deposited in the different regions of the respiratory tract with standard deviation which increases as the number of aerosol particles decreases (DAMA $5 \mu \mathrm{m}, \sigma_{\mathrm{g}} 2.5$, density 3 , standard worker).

\begin{tabular}{|c|c|c|c|c|c|c|c|c|c|c|}
\hline \multirow{2}{*}{$\begin{array}{l}\text { Exposition } \\
\text { (LAI) }\end{array}$} & \multicolumn{10}{|c|}{ Région du tractus respiratoire } \\
\hline & $\mathrm{ET}_{1}$ & $\mathrm{ET}_{2}$ & $\mathrm{ET}_{\mathrm{seq}}$ & $\mathrm{BB}_{1}$ & $\mathrm{BB}_{2}$ & $\mathrm{BB}_{\mathrm{seq}}$ & $\mathrm{bb}_{1}$ & $\mathrm{bb}_{2}$ & $\mathrm{bb}_{\mathrm{seq}}$ & AI \\
\hline 0,5 & $\begin{array}{c}6,4 \times 10^{3} \\
\pm 1 \%\end{array}$ & $\begin{array}{c}7,5 \times 10^{3} \\
\pm 1 \%\end{array}$ & $\begin{array}{c}3,7 \\
+51 \%\end{array}$ & $\begin{array}{c}3,6 \times 10^{2} \\
\pm 6 \%\end{array}$ & $\begin{array}{c}3,6 \times 10^{2} \\
\pm 6 \%\end{array}$ & $\begin{array}{c}5,0 \\
+44 \%\end{array}$ & $\begin{array}{c}1,4 \times 10^{3} \\
\pm 3 \%\end{array}$ & $\begin{array}{c}1,4 \times 10^{3} \\
\pm 3 \%\end{array}$ & $\begin{array}{c}2,0 \times 10^{1} \\
\pm 23 \%\end{array}$ & $\begin{array}{c}1,4 \times 10^{4} \\
\pm 0,8 \%\end{array}$ \\
\hline 0,05 & $\begin{array}{c}6,4 \times 10^{2} \\
\pm 4 \%\end{array}$ & $\begin{array}{c}7,5 \times 10^{2} \\
\pm 4 \%\end{array}$ & $\begin{array}{l}0,35 \\
\mathrm{NA}\end{array}$ & $\begin{array}{c}3,6 \times 10^{1} \\
\pm 17 \%\end{array}$ & $\begin{array}{c}3,6 \times 10^{1} \\
\pm 17 \%\end{array}$ & $\begin{array}{c}0,5 \\
+107 \%\end{array}$ & $\begin{array}{c}1,4 \times 10^{2} \\
\pm 9 \%\end{array}$ & $\begin{array}{c}1,4 \times 10^{2} \\
\pm 9 \%\end{array}$ & $\begin{array}{c}2 \\
+70 \%\end{array}$ & $\begin{array}{c}1,4 \times 10^{3} \\
\pm 3 \%\end{array}$ \\
\hline
\end{tabular}

mieux interpréter les données biologiques. Or en pratique, tous les paramètres caractérisant l'exposition ne sont jamais réellement connus.

Une autre source d'incertitudes a été longtemps négligée. Il s'agit du nombre de particules que renferme l'aérosol. En effet, le modèle de dépôt de la publication 66 de la CIPR (ICRP, 1994) est appliqué de manière déterministe, c'est-à-dire que l'aérosol est supposé renfermer une infinité de particules. Dans le cas d'aérosols renfermant des actinides de forte activité spécifique et/ou de DAMA élevé, le nombre de particules présentes peut être limité. Par exemple, en utilisant les paramètres recommandés par défaut, une exposition de $1 \mathrm{LAI}$ à un aérosol de $\mathrm{PuO}_{2}$

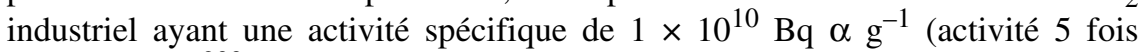
supérieur au ${ }^{239} \mathrm{PuO}_{2}$ ), correspond à environ $2 \mathrm{kBq}$, l'aérosol renfermant en moyenne $2 \times 10^{5}$ particules. Afin d'évaluer le nombre de particules déposées au sein des différentes régions source de l'irradiation du tractus respiratoire, une application stochastique du modèle de dépôt à été développée. Cette approche est basée sur l'exposition à un aérosol simulé dont les particules, de différentes tailles, ont la même probabilité d'être inhalé. Pour chacune de ces particules, la fraction déposée le long du tractus calculée selon le modèle de la publication 66 de la CIPR (ICRP, 1994) est considérée comme une probabilité de dépôt (Fritsch, 2004). Le tableau III compare les dépôts en termes de nombre de particules, avec les incertitudes associées $(\sigma)$, pour une exposition à 0,5 et 0,05 LAI.

Après application stochastique du modèle, les distributions des dépôts en activité et en nombre correspondent à des lois normales, alors qu'après une application déterministe, la distribution en activité est log normale. Cette différence est expliquée du fait que l'approche stochastique prend en compte à la fois l'activité et le nombre de particule, alors que l'approche déterministe ne prend en compte que l'activité. 


\section{TABLEAU IV}

Variabilité des dépôts sous régionaux et de leur épuration par transport des particules. Valeurs issues de Bloch et al. (2003) ; *fraction de l'activité totale de ET1, ET2, BB, bb et AI, $* *$ tractus gastro intestinal.

Variability in regional deposition and in their mechanical clearance. Values from Bloch $e t a l$. (2003); *fraction of total activity in ET1, ET2, BB, bb and AI, **gastro intestinal tract.

\begin{tabular}{|c|c|c|c|c|c|}
\hline $\begin{array}{l}\text { Dépôt sous } \\
\text { régionaux }\end{array}$ & $\begin{array}{c}\text { Valeurs } \\
\text { CIPR 66* }\end{array}$ & Calcul et variabilité & Transport vers & $\begin{array}{c}\text { Fraction transportée } \\
\text { par jour (CIPR 66) }\end{array}$ & Variabilité \\
\hline ET1 & 1 & & l'environnement & 1 & $\sigma_{\mathrm{g}} 1,73$ \\
\hline ET2 & 0,9995 & 1-ETseq & TGI** & 100 & $\sigma_{\mathrm{g}} 1,73$ \\
\hline ETseq & 0,0005 & $\sigma_{\mathrm{g}} 1,73$ & LNet & 0,001 & $\sigma_{\mathrm{g}} 1,73$ \\
\hline BB1 & $\begin{array}{l}\text { BB2/BB varie } \\
\text { en fonction } \\
\text { du } d_{a e}\end{array}$ & & ET2 & 10 & $\sigma_{\mathrm{g}} 1,22$ \\
\hline BB2 & & & ET2 & 0,03 & $\sigma_{\mathrm{g}} 1,73$ \\
\hline BBseq & 0,007 & $\sigma_{\mathrm{g}} 1,73$ & LNth & 0,01 & $\sigma_{\mathrm{g}} 1,73$ \\
\hline bb1 & $\begin{array}{c}\text { bb1/bb2 varie } \\
\text { en fonction } \\
\mathrm{du}_{\mathrm{ae}}\end{array}$ & & BB1 & 2 & $\sigma_{\mathrm{g}} 1,41$ \\
\hline $\mathrm{bb} 2$ & & & BB1 & 0,03 & $\sigma_{\mathrm{g}} 1,73$ \\
\hline bbseq & 0,007 & $\sigma_{\mathrm{g}} 1,73$ & LNth & 0,01 & $\sigma_{\mathrm{g}} 1,73$ \\
\hline AI1 & 0,3 & $\sigma_{\mathrm{g}} 1,10$ & bb1 & 0,02 & constant \\
\hline $\mathrm{AI} 2$ & 0,6 & AI-AI1-AI3 & bb1 & 0,001 & $\sigma_{\mathrm{g}} 1,41$ \\
\hline $\mathrm{AI} 3$ & 0,1 & constant & $\begin{array}{c}\text { bb1 } \\
\text { LNth }\end{array}$ & $\begin{array}{c}0,0001 \\
0,00002\end{array}$ & $\begin{array}{l}\sigma_{\mathrm{g}} 1,73 \\
\sigma_{\mathrm{g}} 1,41\end{array}$ \\
\hline
\end{tabular}

\section{Incertitudes sur le devenir biologique des dépôts}

Le devenir des radionucléides déposés après inhalation est contrôlé par 3 principaux mécanismes: (1) leur transport, (2) leur dissolution, et (3) leur comportement une fois transférés dans le sang sous forme soluble. La publication 66 de la CIPR (ICRP, 1994) considère 2 nouvelles régions pour décrire le transport des particules : les nœuds lymphatiques thoraciques (LNth) et extra thoraciques (LNet). Ces régions sont alimentées par les régions séquestrées (ETseq, BBseq et bbseq) et par une fraction de AI3. Les incertitudes sur la valeur des dépôts et de leur transport ont été évaluées par Bolch et al. (2003). Le tableau IV montre les valeurs que ces auteurs ont rapportées.

L'analyse des mesures expérimentales de la dissolution de différents oxydes $\left(\mathrm{UO}_{2}, \mathrm{U}_{3} \mathrm{O}_{8}, \mathrm{PuO}_{2}\right)$ obtenus à l'aide de divers procédés industriels a montré des incertitudes relativement importantes. Ainsi, la variabilité du taux de dissolution lente $\left(\mathrm{s}_{\mathrm{s}}\right)$ serait décrite par un $\sigma_{\mathrm{g}}$ de l'ordre de 3 (Bolch et al., 2001). En revanche, 


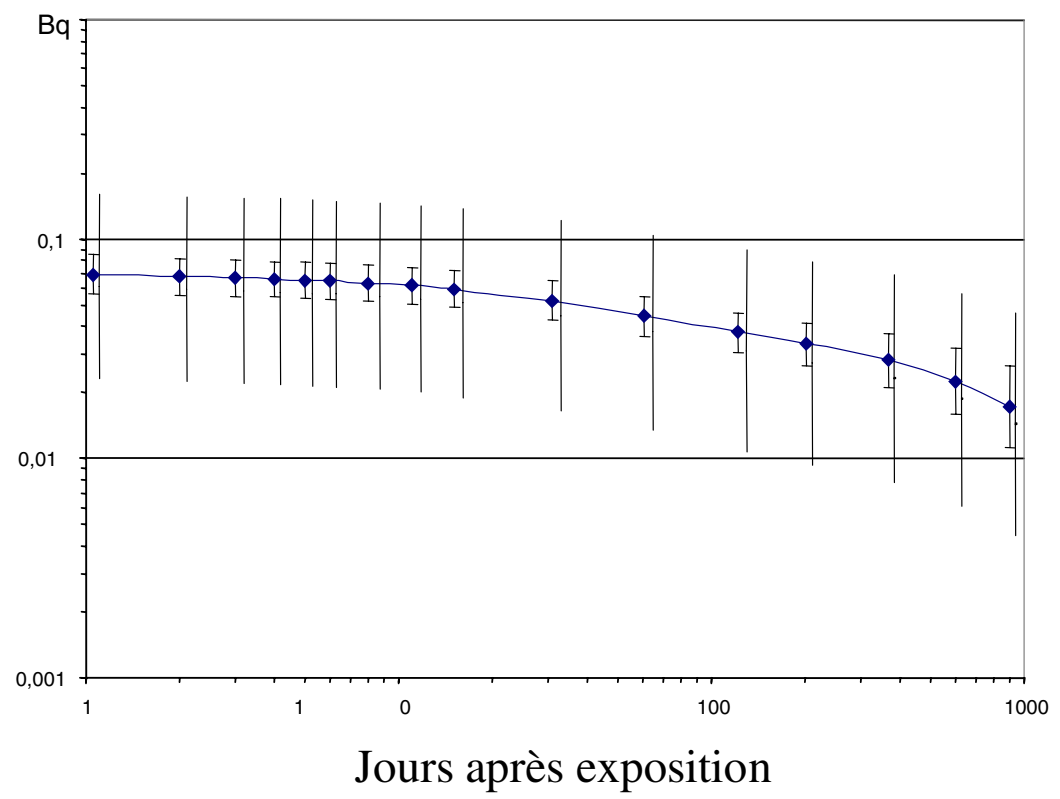

Figure 3 - Intervalle de confiance $95 \%$ de la rétention pulmonaire d'un composé de type S durant les 1000 premiers jours suivant une exposition à un aérosol renfermant $1 \mathrm{~Bq}$. Les barres sans tirets correspondent aux incertitudes lorsque le dépôt initial demeure inconnu, les barres avec tirets lorsqu'il a pu être mesuré.

95\% confidence interval of the pulmonary retention of a type $S$ compound for the first 1000 days following an inhalation exposure to 1 Bq. Simple bars: unknown initial pulmonary deposition, others: initial deposition measured.

pour des composés obtenus à l'aide d'un même procédé, elles apparaissent beaucoup plus faibles ( $\mathrm{s}_{\mathrm{s}}: \sigma=20-30 \%$ ) (Ramounet-Le Gall et al., 2003). Cette dernière variabilité a été appliquée dans notre étude. Enfin, pour le devenir systémique du plutonium dissout qui est décrit par le modèle recyclant de la publication 67 de la CIPR (ICRP, 1993), l'application d'une loi log-normale, ayant un $\sigma_{\mathrm{g}}$ de 1,75, aux différentes constantes de temps décrivant le transfert d'un compartiment vers un autre, à été rapportée pour décrire la variabilité de l'excrétion urinaire du plutonium (Luciani et al., 2003). Cette variabilité a été appliquée dans notre étude.

La figure 3 compare les incertitudes sur l'évolution de la rétention pulmonaire du Pu après inhalation de ${ }^{239} \mathrm{PuO}_{2}$, chez le travailleur standard, lorsque la rétention est évaluée à partir de l'activité des aérosols ou à partir de la mesure de la rétention thoracique précoce $(\mathrm{j}+1)$. Dans ce dernier cas, on attribue un $\sigma$ de $10 \%$ à la mesure 


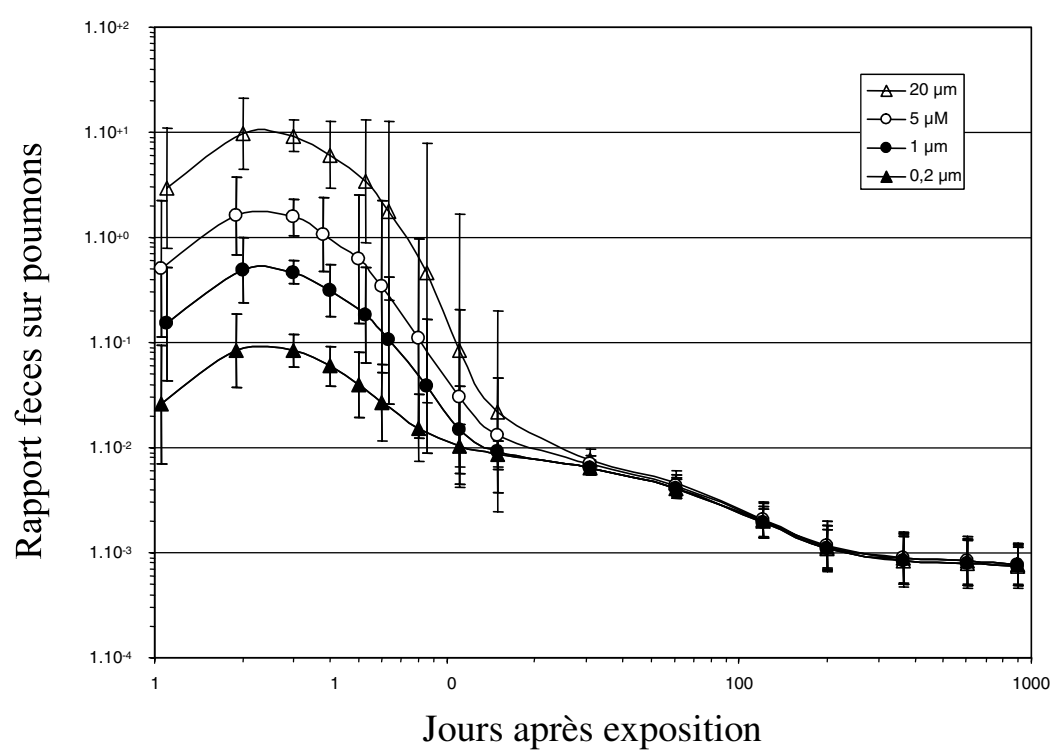

Figure 4-Évolution du rapport de l'activité selles sur poumons en fonction du temps suivant une exposition aigue à un aérosol de type $S$ pour différents DAMA. Barres intervalle de confiance $95 \%$.

Evolution of the activity ratio of faeces versus lungs as a function of time following an acute exposure to a type $S$ aerosol having different AMAD. Bars: $95 \%$ confidence intervals.

thoracique. Les résultats obtenus montrent que la mesure de la rétention pulmonaire permet de limiter de manière significative les incertitudes sur la rétention pulmonaire des actinides durant les 3 premières années suivant la contamination. En revanche, connaissant ou non le dépôt précoce, les incertitudes sur l'excrétion urinaire sont similaires $\left(\sigma_{\mathrm{g}}\right.$ de l'ordre de 2$)$.

La figure 4 montre, en fonction du temps suivant une exposition aiguë, l'évolution du rapport excrétion fécale sur rétention thoracique, pour des composés de type $\mathrm{S}$ ayant différents DAMA.

Bien que durant les 20 premiers jours les incertitudes sur ce rapport soient relativement élevées, il apparaît qu'une évaluation de la taille des aérosols est théoriquement possible durant les 4-5 premiers jours suivant l'exposition. L'analyse de différents cas est en cours pour identifier d'éventuelles expositions à des aérosols très fin (DAMA $<1 \mu \mathrm{m})$.

Divers outils logiciels ont été développés pour permettre l'interprétation des données biologiques et vérifier que la valeur des paramètres introduits dans les modèles pour obtenir le meilleur ajustement des simulations avec les données 

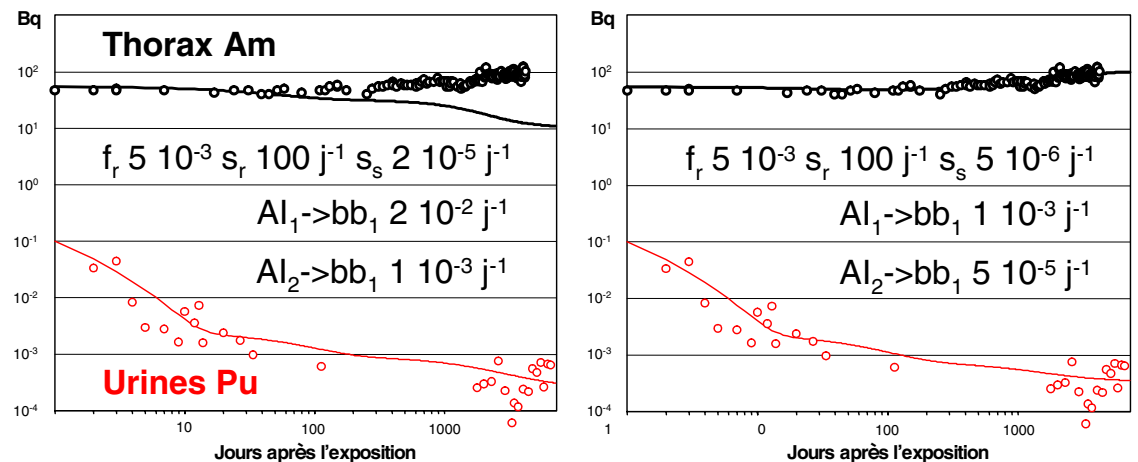

Figure 5-Analyse du cas HAN1. À gauche, les paramètres de l'épuration par transport sont ceux rapportés dans la publication 66 de la CIPR, à droite, leur valeur a été modifiée afin de permettre un bon ajustement des courbes théoriques avec les données biologiques. L'augmentation de l'activité thoracique s'explique par la présence de ${ }^{241} \mathrm{Pu}(1600 \%$ de l'activité $\alpha$ totale au moment de l'exposition).

Analysis of the case HAN1. Left: default parameters of the particle transport model are used, right: specific transport parameters are used to obtain the best fits between theoretical curves and bioassay data. The gradual increase of thoracic activity is due to the presence of ${ }^{241} \mathrm{Pu}$ (1600\% of the total alpha activity at the exposure time).

biologiques est compatible avec les incertitudes qui leur ont été précédemment attribuées. En fait, pour différents cas réels de contamination, la valeur de certains paramètres apparaît être en dehors de l'intervalle de confiance qui a été précédemment rapporté, ou une application déterministe des modèles de la publication 66 de la CIPR (ICRP, 1994) n'est pas possible. À titre d'exemple, l'analyse de 2 cas est ici détaillée.

Le premier cas, HAN1, dont les données biologiques ont été précédemment rapportées (Carbaugh et al., 1991 ; Carbaugh et La Bone, 2003), correspond à une exposition à un aérosol de $\mathrm{PuO}_{2}$ particulièrement peu soluble. La figure 5 montre l'analyse de ce cas.

L'ajustement des données de rétention thoracique de $1{ }^{\text {,241 }} \mathrm{Am}$ et d'excrétion urinaire $\mathrm{du}{ }^{239} \mathrm{Pu}$ à des courbes théoriques ne peut être obtenu qu'en appliquant un taux de dissolution lente $\left(\mathrm{s}_{\mathrm{s}}\right.$ ) égal à $5 \times 10^{-6} \mathrm{j}^{-1}$ (valeur par défaut pour un composé de type $S: 1 \times 10^{-4} \mathrm{j}^{-1}$ ) et en attribuant à la constante de temps de l'épuration par transport des alvéoles $\left(\mathrm{AI}_{1}\right.$ et $\left.\mathrm{AI}_{2}\right)$ vers la lumière des bronchioles des valeurs 20 fois inférieures à celles de la publication 66 de la CIPR (ICRP, 1994), en dehors de l'intervalle de confiance $95 \%\left(\mathrm{AI}_{2} \rightarrow \mathrm{bb}_{1}: \sigma_{\mathrm{g}}=1,41\right)$ rapporté dans la littérature (Bolch et al., 2003). 


\section{TABLEAU V}

Résultat de 10000 simulations pour un travailleur par défaut obtenu après une application stochastique du modèle de dépôt des aérosols de la CIPR 66 (Fritsch, 2004). L'aérosol est constitué de 100 particules. Les incertitudes $(\sigma)$ sur la fraction déposée et le nombre de particules présent au sein de chaque région du tractus respiratoire sont considérables.

Result of 10000 simulations for a standard worker obtained after a stochastic application of the deposition model of ICRP 66 (Fritsch, 2004). The aerosol contains 100 particles. Larges uncertainties in deposited fraction and particle number expressed as standard deviation $(\sigma)$ are observed in each respiratory tract region.

\begin{tabular}{ccccc} 
& activité $\%$ & $\sigma$ & nombre & $\sigma$ \\
\hline $\mathrm{ET}_{1}$ & 30 & 25 & 6,4 & 2,4 \\
$\mathrm{ET}_{2}$ & 38 & 26 & 7,5 & 2,6 \\
$\mathrm{ET}_{\text {seq }}$ & $1,9 \times 10^{-4}$ & $7,5 \times 10^{-3}$ & $4,0 \times 10^{-3}$ & $6,3 \times 10^{-2}$ \\
$\mathrm{BB}_{1}$ & 1,2 & 6,3 & 0,4 & 0,6 \\
$\mathrm{BB}_{2}$ & 0,9 & 4,8 & 0,4 & 0,6 \\
$\mathrm{BB}_{\text {seq }}$ & $9,2 \times 10^{-5}$ & 0,4 & $4,8 \times 10^{-3}$ & $6,9 \times 10^{-2}$ \\
$\mathrm{bb}_{1}$ & 0,8 & 4,5 & 1,4 & 1,2 \\
$\mathrm{bb}_{2}$ & 0,7 & 4,0 & 1,4 & 1,2 \\
$\mathrm{bb}_{\text {seq }}$ & $5,8 \times 10^{-5}$ & 0,2 & $2,0 \times 10^{-2}$ & 0,14 \\
$\mathrm{AI}$ & 9,2 & 12,2 & 14,3 & 3,5 \\
total & 81 & 17 & 31,8 & 4,7 \\
\hline
\end{tabular}

Le second cas illustre les limites d'une application déterministe des modèles de la CIPR lorsque l'aérosol responsable de la contamination renferme un nombre limité de particules (Fritsch et al., 2006a). Dans cet exemple, l'activité spécifique très élevé du composé $\left(1,8 \times 10^{12} \mathrm{~Bq} \mathrm{~g}^{-1}\right)$, près de 1000 fois supérieur à celle $\mathrm{du}$ ${ }^{239} \mathrm{PuO}_{2}$, est liée à la présence de ${ }^{244} \mathrm{Cm}$ et de ${ }^{241} \mathrm{Am}$. Comme le montre le tableau $\mathrm{V}$, l'activité thoracique précoce, mesurée 1 jour après la contamination résulterait pour un aérosol par défaut (DAMA $5 \mu \mathrm{m}, \sigma_{\mathrm{g}}=2,5$ ) de la présence d'une vingtaine de particules.

Dans ce second cas, l'évolution de la rétention thoracique de $1{ }^{241} \mathrm{Am}(63 \mathrm{~Bq}$ à j+0, 33 Bq à j+1, 25 Bq à j+4, 14 Bq à j+6 et <10 Bq au-delà) est incompatible avec les modèles biocinétiques de la CIPR. En revanche, elle peut être expliquée en tenant compte du devenir des quelques particules qui renferment l'essentiel de l'activité. Cette hypothèse est étayée par le fait que les différents comptages thoraciques positifs ont montré, de manière systématique, une importante variabilité des mesures et des spectres obtenus à l'aide de chacun des 4 détecteurs. Ce cas constitue un exemple dans lequel le concept de la DPUI n'est pas applicable, une dose réaliste ne pouvant être évaluée qu'à partir d'une déconvolution des données biologiques. 


\section{Incertitudes sur les doses}

Les incertitudes sur les doses équivalentes pulmonaires ont été évaluées pour des aérosols monodispersés de ${ }^{239} \mathrm{PuO}_{2},{ }^{238} \mathrm{UO}_{2}$ et ${ }^{238} \mathrm{U}_{3} \mathrm{O}_{8}$. Elles sont exprimées sous forme de rapport 95 sur $5 \%$. Elles concernent, tout d'abord, le nombre de rayonnements alpha émis dans les différentes régions source (Bolch et al., 2003), puis les doses équivalentes, en tenant compte d'une variabilité de l'épaisseur de différentes régions, du poids des régions cibles et du risque relatif d'apparition de cancer selon les régions (Farfan et al., 2003). Les incertitudes sur le nombre de désintégrations et sur les doses apparaissent sensiblement équivalentes, voisines de celles rapportées précédemment pour le dépôt des aérosols (Bolch et al., 2001). Ceci s'explique par la non additivité des incertitudes et l'interdépendance de nombreux paramètres. Pour un diamètre compris entre 0,1 et $2 \mu \mathrm{m}$, les rapports $95 \%$ / $5 \%$ sont voisins de 10, sauf pour les bronches et les nœuds lymphatiques thoraciques, où ils sont beaucoup plus élevés. Ils augmentent graduellement audelà, pour atteindre des valeurs comprises entre 100 et 1000, lorsque le diamètre aérodynamique est égal à $10 \mu \mathrm{m}$.

Nous avons entrepris une démarche analogue pour des aérosols polydispersés de ${ }^{239} \mathrm{Pu}$. Aujourd'hui, la variabilité des paramètres cytologiques et anatomiques rapportée par Bolch et al. (2003) n'est pas encore prise en compte. Il faut noter que l'épaisseur des différentes régions cibles rapportée par Farfan et al. (2003) est très différente de celles rapportées dans la publication 66 de la CIPR (ICRP, 1994). Les études ont concerné tout d'abord des composés modérément (type M) ou faiblement soluble (type $\mathrm{S}$ ), en calculant également les doses équivalentes thoraciques et extra thoraciques, mais aussi les DPUI. Pour le type $\mathrm{S}$ et des DAMA entre 0,1 et $50 \mu \mathrm{m}$, les rapports sont sensiblement constants : 6 et 4 pour les doses équivalentes extra thoraciques et thoraciques, et 4 pour les DPUI. Pour le type M, ces valeurs varient respectivement entre 9 et 100 , entre 4 et 12 et entre 2 et 5. D'une manière générale, l'utilisation de facteurs de pondération pour le calcul des doses équivalentes tend à diminuer les incertitudes.

La figure 6 compare, pour le ${ }^{239} \mathrm{PuO}_{2}$, et pour le DAMA par défaut, les incertitudes sur les DPUI et les doses « efficaces » délivrées aux principaux tissus cibles, connaissant ou non le dépôt pulmonaire précoce (Fritsch, 2005).

La connaissance des dépôts thoraciques précoces permet de diminuer d'un facteur 3 à 4 les incertitudes sur les doses thoraciques et les DPUI.

\section{Conclusion et perspectives}

Cette synthèse, non exhaustive, montre l'intérêt de la connaissance des incertitudes sur les paramètres physiques décrivant les aérosols, et de la variabilité 


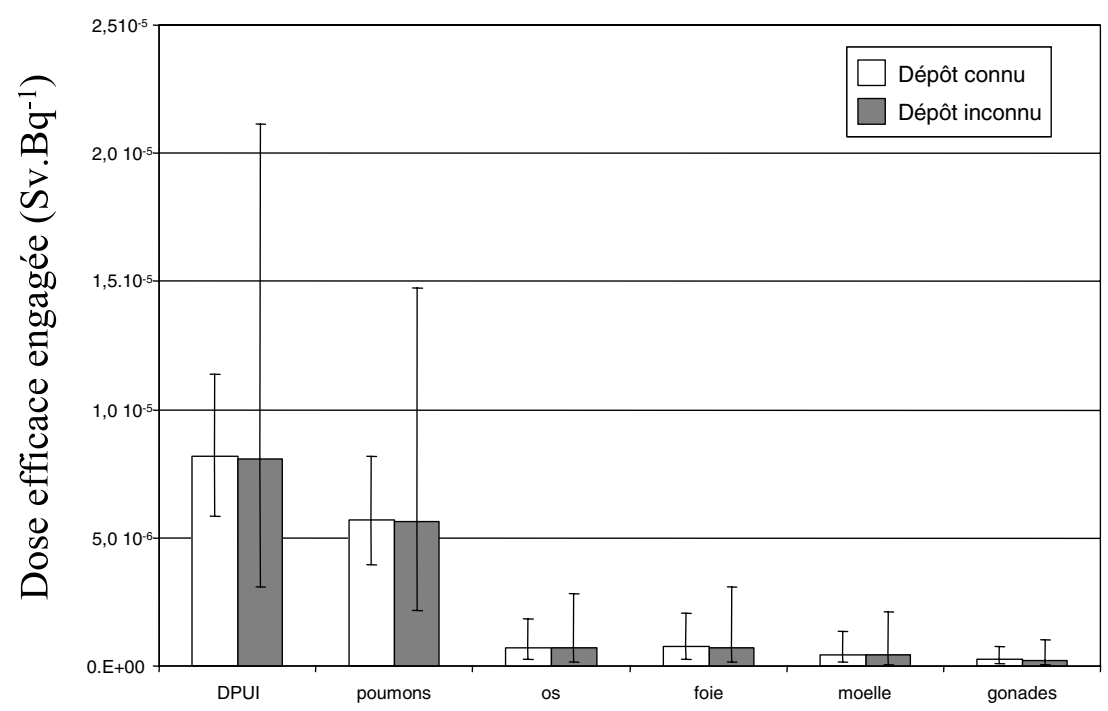

Figure 6 - Comparaison des incertitudes sur les doses efficaces engagées et les DPUI lorsque le dépôt des aérosols peut être ou non mesurable. Résultat de 1000 simulations effectuées pour un aérosol de ${ }^{239} \mathrm{PuO}_{2}$ en prenant les valeurs par défaut recommandées par la CIPR pour les travailleurs. Les barres représentent les intervalles de confiance $95 \%$. D’après Fritsch (2005).

Comparison of uncertainties in committed effective doses and DPUI when early thoracic deposition is or is not measured. Result of 1000 simulations for ${ }^{239} \mathrm{PuO}_{2}$ using default parameters for standard workers. Bars: 95\% confidence intervals. From Fritsch (2005).

de l'anatomie et de la physiologie des individus pour une meilleure évaluation des expositions, des niveaux de contamination et des DPUI. Les études ne sont pas encore achevées ; elles permettront, à terme, d'associer systématiquement des incertitudes aux différents résultats obtenus après calcul de dose, et, en cas de contamination, de prendre en compte les paramètres propres aux individus.

Après inhalation d'actinides, lorsque seule l'activité des aérosols est accessible, l'intervalle de confiance, exprimé sous forme du rapport $97,5 \% / 2,5 \%$, pour les doses efficaces est de l'ordre de 10. Ceci montre les difficultés d'une hiérarchisation de leur toxicité, à partir des DPUI, en l'absence d'une caractérisation précise des aérosols. En fait, après inhalation de composés ayant une forte activité spécifique, il apparaît que la principale source d'incertitudes réside dans le nombre de particules constituant l'aérosol. Lorsque ce nombre est trop faible, la notion de DPUI devient alors inapplicable (Fritsch et al., 2006a). Un bilan des contaminations internes observées sur le site du Commissariat à l'énergie atomique de Cadarache, durant ces dernières années, 
suggère que ceci serait le cas pour certaines contaminations par inhalation (Blanchin et al., 2004, 2005). Ainsi, pour les faibles niveaux de contamination, l'application déterministe des modèles de la CIPR serait souvent impossible à l'échelle d'un individu. Cependant cette approche pourrait être réaliste à l'échelle d'une population, dans la mesure où l'on tient compte des incertitudes.

La variabilité attribuée aux différents paramètres des modèles peut sembler parfois arbitraire, du fait notamment, de l'insuffisance des données scientifiques disponibles. Ainsi, l'essentiel des données humaines concernant l'épuration par transport intéresse une période limitée à 1 an, après une contamination aiguë par des aérosols. La publication 66 de la CIPR (ICRP, 1994) a attribué une variabilité limitée à la cinétique de l'épuration tardive dont s'est inspiré Bolch et al. (2003). En fait, l'analyse du cas HAN1 montre une variation de cette épuration au-delà de la variabilité attendue. Ce phénomène contribue à une augmentation de la dose efficace de près d'un facteur 4 , par rapport à la dose obtenu après un calcul qui utilise les paramètres par défaut. Un autre cas de contamination présente une inhibition analogue de l'épuration pulmonaire tardive (Fritsch, 2005). Après une contamination significative, il apparaît donc nécessaire d'effectuer un suivi prolongé de la rétention thoracique pour obtenir une dosimétrie suffisamment précise.

Lorsque le niveau de contamination est accessible à la mesure, la connaissance des diverses incertitudes permet de proposer un calendrier de prélèvements biologiques les plus pertinents afin d'assurer un suivi efficace des travailleurs, au cas par cas, en appliquant les recommandations en vigueur. Néanmoins, le résultat de cette démarche réglementaire pourrait ne pas correspondre à une évaluation réaliste de la biocinétique des radiocontaminants. En effet, les modèles utilisés aujourd'hui, ainsi que la valeur de leurs paramètres, sont encore discutés. Le modèle de la publication 67 de la CIPR (ICRP, 1993) décrivant le devenir du plutonium soluble ayant gagné le sang considère des échanges entre différents compartiments qui, physiologiquement, ne peuvent exister. Ainsi, par exemple, une partie du plutonium retenu dans les tissus mous (ST1) migre directement vers la vessie. Ceci permet de décrire une excrétion urinaire réaliste mais pourrait contribuer à une sous estimation de l'activité présente dans le sang et les liquides interstitiels. Par ailleurs, chez l'adulte, d'importantes modifications du métabolisme osseux se produisent au cours du vieillissement, qui ne sont pas prises en compte. Ainsi, l'application de nouveaux modèles plus physiologiques (Leggett et al., 2005) pourrait apparaître utile. Cependant, les doses calculées avec cette approche ne seraient plus réglementaires. Une dernière difficulté est souvent rencontrée lors de l'analyse des données biologiques : les traitements par le DTPA qui augmentent l'excrétion naturelle des éléments transuraniens. Aujourd'hui, les méthodes proposées pour interpréter ces traitements (Hall et al., 1978; 
P. FRITSCH et al.

Piechowski et al., 2003; Bailey et al., 2003) ne permettent pas encore l'interprétation de certains cas où des administrations répétées de l'agent chélateur ont été effectuées.

Différentes recherches sont en cours afin d'améliorer l'interprétation des données biologiques après différents types de contamination. Une première modélisation a été effectuée à partir d'un cas de blessure contaminé par du plutonium, le travailleur ayant reçu des perfusions répétées de DTPA. L'application du modèle systémique de Leggett et al. (2005) apparaît particulièrement utile car elle permet d'obtenir le meilleur ajustement entre les données biologiques et les valeurs calculées (Fritsch et al., 2006b). Une telle approche devrait permettre l'élaboration de protocoles de traitements sur la base d'un bénéfice, en termes de réduction de doses. Par ailleurs, un référentiel didactique est en cours d'élaboration afin de justifier, notamment, l'application déterministe ou stochastique des modèles ascendants et de rendre plus réaliste l'évaluation du risque de cancer (Mièle et al., 2006). Dans ce cadre, la question se pose sur l'assimilation de la dose efficace à un risque de cancer. En effet, des études expérimentales indiquent, qu'après inhalation d'actinides, le risque de cancer est modulé par l'hétérogénéité de la distribution de la dose alpha au sein des poumons (Fritsch et al., 2003a). Une synthèse concernant les relations entre doses et risques de cancer est en cours de préparation.

Remerciements. Les études ont été cofinancées dans le cadre du programme d'intérêt commun CEA/COGEMA, PIC D13.

\section{RÉFÉRENCES}

Aden J., Scott B.R. (2003) Modeling variability and uncertainty associated with inhaled weapons-grade $\mathrm{PuO}_{2}$, Health Phys. 84, 726-736.

Ansoborlo E., Chazel V., Hengé-Napoli M.H., Pihet P., Rannou A., Bailey M.R., Stradling N. (2002) Determination of the physical and chemical properties, biokinetics, and dose coefficients of uranium compounds handled during nuclear fuel fabrication in France, Health Phys. 82, 279289.

Ansoborlo E., Bérard P., Eckerman K.F., Berkovski V., Birchall A., Fry F.A., Guilmette R.A., Miller G., Ishigure N., Lipsztein J.L., Noßke D. (2003) Review of methods and computer codes for bioassay data interpretation, Radiat. Prot. Dosim. 105, 341-346.

Apostoaei A.I., Miller L.F. (2004) Uncertainties in dose coefficients from ingestion of ${ }^{131} \mathrm{I},{ }^{137} \mathrm{Cs}$ and ${ }^{90} \mathrm{Sr}$, Health Phys. 86, 460-482.

Bailey B.R., Eckerman K.F., Townsend L.W. (2003) An analysis of a puncture wound case with medical intervention, Radiat. Prot. Dosim. 105, 509-512.

Blanchin N., Desloires S., Grappin L., Guillermin A.M., Lafon P., Miele A. (2004) Protocoles de prise en charge des incidents d'expositions internes au plutonium dans un service médical d'installation nucléaire de base : élaboration - mise en place - évaluation - validation de 1996 à 2002, Radioprotection 39, 59-76. 
Blanchin N., Fottorino R., Grappin L., Guillermin A.M., Lafon P., Mièle A., Ruffin M. (2005) Surveillance systématique des expositions aux transuraniens par les analyses radiotoxicologiques des selles: retour d'expérience, intérêt et perspectives, Radioprotection 40, 231-243.

Bolch W.E., Farfan E.B., Huh C.H., Huston T.E., Bolch W.E. (2001) Influences of parameter uncertainties within the ICRP-66 respiratory tract model: Particle deposition, Health Phys. 81, 378-394.

Bolch W.E., Huston T.E., Farfan E.B., Vernetson W.G., Bolch W.E. (2003) Influences of parameter uncertainties within the ICRP-66 respiratory tract model: Particle clearance, Health Phys. 84, $421-435$.

Carbaugh E.H., Bihl D.E., Sula M.J. (1991) Long-term follow-up of HAN-1, an acute plutonium oxide inhalation case, Radiat. Prot. Dosim. 38, 99-104.

Carbaugh E.H., La Bone T.R. (2003) Two case studies of highly insoluble plutonium inhalation with implications for bioassay, Radiat. Prot. Dosim. 105, 133-138.

Dorrian M.D., Bailey M.R. (1995) Particle size distributions of radioactive aerosols measured in the workplaces, Radiat. Prot. Dosim. 60, 119-133.

Dunning D.E., Schwarz G. (1980) Variability of human thyroid characteristics and estimates of dose for ingested 131I, Health Phys. 40, 661-675.

Farfan E.B., Huston T.E., Bolch W.E., Vernetson W.G., Bolch W.E. (2003) Influences of parameter uncertainties within the ICRP-66 respiratory tract model: Regional tissue doses for ${ }^{239} \mathrm{PuO}_{2}$ and ${ }^{238} \mathrm{UO}_{2} /{ }^{238} \mathrm{U}_{3} \mathrm{O}_{8}$, Health Phys. 84, 436-450.

Fritsch P. (2004) Uncertainties in doses due to the number of aerosol particles: study on ${ }^{239} \mathrm{PuO}_{2}$ using default parameters for workers, Health Phys. 87, 416-422.

Fritsch P. (2005) Uncertainties in doses calculated according to ICRP recommendations after inhalation of ${ }^{239} \mathrm{PuO}_{2}$ and early chest monitoring. Congrès Individual Monitoring, Vienne, Autriche, avril 2005, Radiat. Prot. Dosim. (sous presse, 2006).

Fritsch P. (2006) Uncertainties in aerosol deposition within the respiratory tract calculated according to ICRP recommendations: a study in adults. Health Phys. 90, 114-126.

Fritsch P., Guillet K. (2002) Granulometry of aerosols containing transuranium elements at the work place: an estimate using autoradiographic analysis, Ann. Occupl. Hyg. 46 (sup1), 292-295.

Fritsch P., Dudoignon N., Guillet K., Oghiso Y., Morlier J.P., Monchaux G. (2003a) Does DPUI calculated for inhaled actinide oxides actually reflect the risk of malignant lung tumour induction? Radiat. Prot. Dosim. 105, 149-152.

Fritsch P., Champion C., Ménétrier F., Delforge J. (2003b) Uncertainties on the committed equivalent dose to the thyroid as a function of age for different iodine isotopes, Radiat. Prot. Dosim. 105, 247-250.

Fritsch P, Raynaud P, Blanchin N and Mièle A. (2006a) Limits of DPUI application associated with the number of particles within actinide aerosols. Présentation au congrès Internal dosimetry of radionuclides, Montpellier, octobre 2006, Radiat. Prot. Dosim. (à soumettre).

Fritsch P., Grappin L., Guillermin A.M., Fottorino R., Ruffin M., Mièle A. (2006b) Modelling of bioassay data from a Pu wound treated by repeated DTPA perfusions: biokinetics and dosimetric approaches. Présentation au congrès Internal dosimetry of radionuclides, Montpellier, octobre 2006, Radiat. Prot. Dosim. (à soumettre).

Hall R.M., Poda G.A., Fleming R.R., Smith J.A. (1978) A mathematical model for estimation of plutonium in the human body from urine data influenced by DTPA therapy, Health Phys. 34, 419-432.

Hamby D.M., Benke R.R. (1999) Uncertainties of the iodine-131 ingestion dose conversion factor, Radiat. Prot. Dosim. 82, 245-256. 
P. FRITSCH et al.

Harvey R.P., Hamby D.M. (2001) Uncertainty in particulate deposition for $1 \mu \mathrm{m}$ AMAD particles in an adult lung model, Radiat. Prot. Dosim. 95, 239-247.

Harvey R.P., Hamby D.M., Benke R.R. (2003) Age-specific uncertainty of the ${ }^{131}$ I ingestion dose conversion factor, Health Phys. 84, 334-343.

ICRP Publication 67 (1993) Age-dependent doses to members of the public from intake of radionuclides: Part 2, Ingestion dose coefficients, Ann. ICRP 23 (3-4).

ICRP Publication 66 (1994) Human respiratory tract model for radiological protection, Ann. ICRP 24 (1-3).

Layton D.W. (1993) Metabolically consistent breathing rates for use in dose assessments, Health Phys. 64, 23-36.

Leggett R.W., Eckerman K.F., Khokhryakov V.F., Suslova B.G., Krahenbuhl F.P., Miller S.C. (2005) Mayak worker study: an improved biokinetic model for reconstructing doses from internally deposited plutonium, Radiat. Res. 164, 111-122.

Luciani A., Doerfel H., Polig E. (2003) Uncertainty analysis of the urinary excretion of plutonium, Radiat. Prot. Dosim. 105, 383-386.

Mièle A., Blanchin N., Raynaud P., Quesne B., Giraud J.M., Fottorino R., Bérard P., Ansoborlo E., Franck D., Blanchardon E., Challeton-de Vathaire C., Piechowski J., Fritsch P. (2006) MEDOR, a didactic tool to support interpretation of bioassay data after internal contamination by actinides. Présentation au congrès Internal dosimetry of radionuclides, Montpellier, octobre 2006, Radiat. Prot. Dosim. (à soumettre).

Piechowski J., Menoux B., Mièle A., Grappin L., Guillermin A.M., Fottorino R., Ruffin M. (2003) Implications du médecin du travail et de l'expert dans la gestion et la dosimétrie d'un incident de contamination : exemple d'une plaie contaminée par le plutonium, Radioprotection 38, 29-50.

Ramounet-Le Gall B., Fritsch P., Abram M.C., Rateau G., Grillon G., Guillet K., Baude S., Bérard P., Ansoborlo E., Delforge J. (2002) Mesure de paramètres spécifiques pour le calcul de dose après inhalation d'aérosols renfermant des éléments transuraniens, Can. J. Physiol. Pharmaco. 80, 727-732.

Ramounet-Le Gall B., Rateau G., Abram M.C., Grillon G., Ansoborlo E., Bérard P., Delforge J., Fritsch P. (2003) In vivo measurement of Pu dissolution parameters of MOX aerosols and related uncertainties on DPUI values, Radiat. Prot. Dosim. 105, 153-156. 